\title{
VALORACIÓN AMBIENTAL PARA LA GENERACIÓN DE UN MODELO PARTICIPATIVO DE EDUCACIÓN NO FORMAL
}

\author{
Jorge Villadiego Lorduy ${ }^{1}$ \\ Dennis Huffman-Schwocho ${ }^{2}$ \\ Stalyn Guerrero Gómez ${ }^{3}$ \\ Sandra Rivero Espitia ${ }^{4}$ \\ Adolfo Cortecero Bossio ${ }^{5}$
}

Recibido el 22 de julio de 2014, aprobado el 6 de diciembre de 2014 y actualizado el 30 de abril de 2015

DOI: 10.17151/luaz.2015.41.10

\section{RESUMEN}

El propósito de este artículo es establecer un diagnóstico de los humedales de Bañó y $L$ os Negros basado en el análisis y caracterización de las esferas social, económica y natural, a fin de entender las causas de lo s eventos físicos, económicos, sociales y educativos qu e expliquen el desfa samiento entre percepciones y cultura $s$ adecuadas para la sostenibilidad ambiental del territorio y la consideración de tales causas para la generación de un modelo educativo no formal. La problemática ambiental existente en lo $s$ humedales es el re sultado de la $s$ presiones de tipo ant rópico a la que han sido sometidos, la ausencia de una cultural ambiental ha llevado a sus habitantes a desequilibrar tales ecosistemas. Como resultado del diagnóstico territorial se identifica ron una serie de problemas ambientales: desecación de hume dales, pérdida de la biodiversida $d$, deficientes condiciones sanitarias, contaminación del suelo y agua, y manejo inadecuado de residuos. Factores como el bajo nivel educativo, las e scasas oportunidades de empleo y lo s pocos ingresos por núcleo familiar inciden de forma indirecta en el deterioro de los humedales. Se resalta que un $85,8 \%$ del total de la po blación tiene más de 16 años de vivir en el área de estudio, por lo tanto tienen un cono cimiento empírico de los humedales y de los problemas presentes en ellos.

\section{PALABRAS CLAVE}

Perfil ambiental, problemas ambientales, pobreza, bajo nivel de educación, precarias condiciones socioeconómicas.

\section{ENVIRONMENTAL ASSESSMENT FOR THE GENERATION OF A PARTICIPATORY MODEL OF NON FORMAL EDUCATION}

\section{ABSTRACT}

The purpose of this article is to establish a diagnosis of the wetlands of Bañó an d Negros based on the analysis and characterization of the so cial, economic and natural spheres in order to understand the causes of physical, economic, social and educational events th at explain the $d$ estaging between 
perceptions and cultures adequate for the environm ental sustainability of the territory and the consideration of such causes for the gen eration of a non-formal educational system. The existing environmental problem in th e wetlands of Bañó and Negros is the result of the pressures of anthropic type they have been submitted to, the absen ce of an environmental culture has led residents to unbalance the ecosystems in mention. As a result of the territorial diagn osis, a number of environmental problems ranging from desiccation of wetlands, loss of biodiversity, poor sanitary conditions, soil and water pollution to inappropriate waste management were ide ntified. Factors such a s a low level of education, limited employment opportunities and little hou sehold income affect indirectly the deteri oration of wetlands. It's important to note that $85,8 \%$ of the to tal population has been living in the study area for more than 16 years. Therefore they have $a b$ road knowledge of the wetla nds and of the proble ms present in them.

\section{KEY WORDS}

Environmental profile, environmental problems, poverty, low education, poor socio-economic conditions.

\section{INTRODUCCIÓN}

Hoy día la gl obalización económica impone nuevas pautas para la producción y consum o de recursos. Para Me ira (2006) la relación entre globalización y crisis ambiental se puede sintetizar en una serie de fenómenos que, en ri gor, no son nuevos pero que en las últimas dos décadas se han agudizado y acelerado. Hay una estrecha relación entre las sociedades industrializadas y el deterior ambiental a escala global, Capra (2003) afirma que desde las últimas dé cadas del siglo XX las actividade $s$ económicas están d eteriorando la bio sfera y la vida huma na, hasta el punto de llegar a causar daños irreversibles. El actual modelo económico conlleva a un consumo acelerado de recursos y a la ge neración de grandes volúmenes de residuos, en ambos casos se ge neran impactos negativos al ambie nte. Al respe cto Bindé (2008, p. 104) señala: "No se pueden oponer, desarrollo sostenible y desarrollo a secas, lucha contra la pobreza y conservación de los ecosistemas", por el contrario, es necesario "luchar en todos los frentes a la vez: a partir de ahora, el enfoque debe ser global, lo mismo que la conciencia".

Colombia no es ajena a la crisis ambiental, sobre todo para los ecosistemas tipo humedal donde cada vez se van desecand o y deteriorando más sus condiciones ambientales. La región Caribe presenta un ambiente de aguas saladas y salobres que permite la presencia, en esta zo na, de la mayor varia ción en cuanto a humedales costeros del país se refiere, ellos son: ribereños, lacustres, palustres, marinos y estuarios; de amplias playas arenosas y fondos lodosos. Forman parte de estos ecosistemas la Ciénaga Grande del Magdalena, la desembocadura de los ríos Sinú y Atrato y la Bahía de Ca rtagena. La extensión territorial va 
desde los límites con Panamá hasta los límites con Venezuela (aproximadamente $142.000 \mathrm{~km}^{2}$ ). Comprende localidades de los departamentos de La G uajira, Magdalena, Atlántico, Bolívar, Sucre, Córdoba y Antioquía entre $7^{\circ} 56^{\prime}$ y $12^{\circ} 25^{\prime}$ latitud Norte y $77^{\circ} 20^{\prime}$ y $71^{\circ} 08^{\prime}$ longitud Oeste (Rangel, Lowy y Aguilar, 19 97). En el caso de los humedales de Bañó y Los Negros (ubicados en la región del bajo Sinú, departamento de Córdoba) debido a la presión que ejercen sus habitantes lo están llevando a sus límites ecológicos, lo anterior, originado por la demanda de recursos naturales. Por ello y con el objetivo de dar una solución a los problemas ambientales se desarrolló una valoración ambiental de los espejos de agua y sus po blaciones vecinas con el fin de conocer el estado real en que se encuentran.

La ausencia de una cul tura ambiental que ge nere acciones positivas por parte de los habitantes de estos humedales hacia el medio, los bajos ingresos económicos y de nivel de educación son factores que están influyendo en su degradación. El fin de la valoración ambiental fue identificar ciertos elementos que se pudieran tener en cuenta a la hora de g enerar un m odelo educativo no formal pa ra la mitiga ción de lo $\mathrm{s}$ problemas ambientales de este territorio en particular.

La educación ambiental (EA) hoy día se ha convertido en un poderoso instrumento de la gestión ambiental y debe entenderse como un proceso dinámico y participativo, que busca despertar en la población una conciencia que le permita identificarse con la problemática ambiental tanto a nivel general, co mo a nivel especifico. Para poder formular modelos educativos es necesario diagnosticar el territorio en las e sferas social, económica y natural; esto proporcionará una radiografía del territorio y a partir de un a nálisis profundo empezar a re conocer ciertas particularidades que permitan generar y arti cular modelos educativos aplicados al as poblaciones que habitan áreas aledañas a humedales o ecosistemas de este tipo.

\section{MATERIALES Y MÉTODOS}

El método que se utili zó en el est udio de la $s$ condiciones ambientales de los humedales de Bañó y Los Negros se soporta en el análisis de información primaria y secundaria. Primero se buscó identificar las características del territorio para después establecer relaciones de causa-efecto sobre el estado actual del mismo. En términos de información secundaria se recopiló información de proyectos ambientales desarrollados en el área de estudio. Para el estudio de la información primaria se diseñó y aplicó un i nstrumento de investigación tipo cuestionario, que buscó recopilar información de va riables socioeconómicas, y problemas y conflictos ambientales, que permitiera contrastar con la información secundaria analizada y con las visitas a campo realizadas a los ecosistemas en mención.

La población universo de la investiga ción está determinada por 645 viviendas habitadas pertenecientes a los corregimientos de 
Cotocá Arriba (110), Castilleral (146) y Palo de Agua (389). Se determinó un diseño de muestreo por estrato, donde los estratos corresponden a los diferentes corregimientos, para Cotocá Arriba y Castilleral se apli có un muestreo sistemático y en el caso de Palo de Agua se implementó un muestreo aleatorio simple. Se aplicaron un total de 72 encuestas con 40 ítems por cuestionario, las encuestas se distribuyeron de la siguiente forma: Cotocá Arriba y Castilleral 12 cada una, Palo de Agua 48. Los diseños implementados fueron seleccionados debido a que eran los que mejor se ajustaban a la organi zación espacial de lo $\mathrm{s}$ corregimientos y a la limitación de recursos disponibles.

Para el procesamiento y análisis de la información recolectada del cuestionario se emplearon métodos de estadística inferencial basada en cálculo de estimaciones porcentuales y valore $\mathrm{s}$ absolutos. La información del total de viviendas existentes a la fecha y sobre la cual se trabajó el diseño muestral se obtuvo de los líderes comunitarios de cada uno de los pueblos y de la verificación en campo de las viviendas habitadas. El software con el que se tra bajó el análisis estadístico fue $R$ versió n 3.0.2. Se asoció como técnica el trabajo de campo para la verificación de la problemática ambiental existente en los espejos de agua.

Por último, el área de e studio se encuentra delimitada entre las siguientes coordenadas: 9 ${ }^{\circ} 7^{\prime} 22,14^{\prime \prime} \mathrm{N}-75^{\circ} 52^{\prime} 30,68^{\prime \prime} \mathrm{W}, 9^{\circ} 9^{\prime} 1,08^{\prime \prime}$

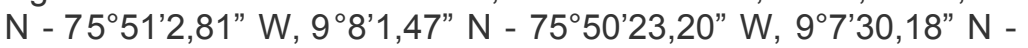
$75^{\circ} 50^{\prime} 2,28^{\prime \prime}$ W y $9^{\circ} 6^{\prime} 50,00^{\prime \prime} \mathrm{N}-75^{\circ} 50^{\prime} 42,97^{\prime \prime} \mathrm{W}$.

\section{RESULTADOS Y DISCUSIÓN}

\section{Los humedales de Bañó y Los Negros y sus características físico-bióticas}

Los humedales de Bañó y Los Negros se encuentran ubicados en el bajo Sinú, exactamente entre los corregimientos de Cotocá Arriba, Castilleral y Palo de Agua, todos pertenecientes al área rural del $m$ unicipio de Lorica, departamento de Córdoba, Colombia. La primera ciénaga está localizada a $09^{\circ} 08^{\prime} 09^{\prime \prime}$ N y $075^{\circ} 50^{\prime} 12^{\prime \prime \prime}$ W, la segunda a $09^{\circ} 07^{\prime} 59^{\prime \prime \prime} \mathrm{N}$ y $075^{\circ} 50^{\prime} 49^{\prime \prime \prime}$ W. (Ver Figura 1). 


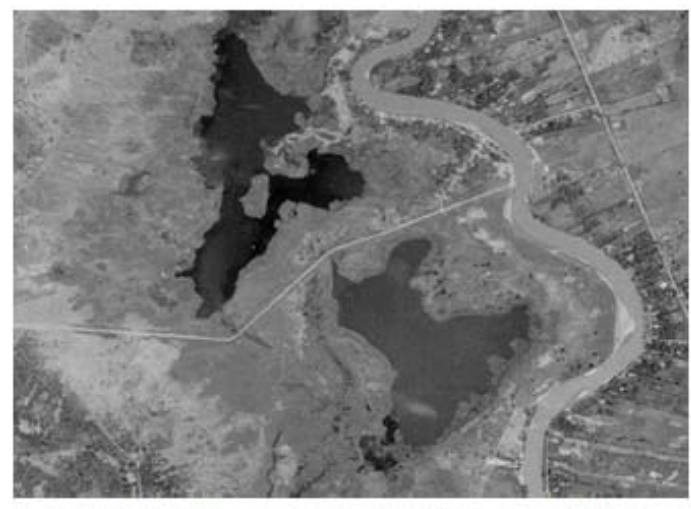

Fuente: Instituto Geográfico Agustín Codazzi (1994).

Figura 1. Fotografía aérea de las ciénagas de Bañó y Los Negros, margen izquierdo del río Sinú, en el corregimiento de Cotocá Arriba, municipio de Lorica. Ciénaga de Bañó (parte inferior en la imagen) y ciénaga Los Negros (parte superior en la imagen).

La geología de los h umedales de Bañó y Lo s Negros se enmarcan en el valle del río Sinú correspondiendo al peri odo cuaternario, formándose una planicie fluvio-lacustre, que comprende diques naturales, basines, complejos de esto $\mathrm{s} y$ valles estrechos. Dentro de la planicie existe una serie de terrazas bajas, compuestas generalmente por materiales finos, tales como arcillas, li mos y arenas finas, que son poco permeables y facilitan I os encharcamientos en épocas de muchas lluvias, agrietándose en los períodos de pocas lluvias. El área occidental del valle del Sinú, que comprende los municipios de Cereté, San Pelayo, Lorica y San Berna rdo del Viento, tiene en su parte baja intercalaciones de arcillas, areniscas, arcillolitas y conglomerados, y en su parte alta ni veles calcáreos y are nas con incrustaciones de moluscos que corresponden al Mioceno superior y Pleisto ceno (Instituto Geo gráfico Agustín Codazzi , 1983).

Adicionalmente, los suel os de los humedales en cuestión pertenecen a la planicie fluvio-lacustre del Sinú, de la cual forma parte el área de influencia de los mismos, presenta suelos desarrollados a pa rtir de materiales medios, finos y g ruesos, preferencialmente ácidos. El río Sinú y su $s$ afluentes han depositado sedimentos en las á reas más bajas de la llanu ra aluvial, tiene un relieve plano y plano-cóncavo, con pendientes de $0-3 \%$ y soportan inundaciones periódicas (Corporación de los Valles del Sinú y San Jorge, 2002).

En cuanto a la zo na de vida, características climáticas y de precipitación la zona ale daña a lo s humedales, se en cuentra clasificada como bo sque seco Tropical (bs-T), según la clasificación de zonas de vida de L. Holdridge, con temperaturas que oscilan entre 26 y $28,5^{\circ} \mathrm{C}$ y p recipitaciones entre 1000 y $1500 \mathrm{~mm}$ anuales. Las ciénagas de Bañó y Los Negros cuentan con una precipitación promedio anual de $1300 \mathrm{~mm}$ y una temperatura de $28^{\circ} \mathrm{C}$ (Corporación de los Valles del Sinú y San Jorge, 2002). Además, una humedad relativa promedio anual de 
$84 \%$ y un valor medio anual de brillo solar representado en 2030 horas (Alcaldía de Lorica, 2001).

En términos de fauna, en los e spejos se puede señalar gran cantidad de aves, entre estas las especies Pisingo (Dendrocygna autumnalis) y el Pato viuda ( Dendrocygna viduata), con mayor densidad poblacional, igualmente Garza parda (Ardea herodias), Garza blanca real (Casmerodius albus), Garcita blanca patiamarilla (Egretta thula), Garcipolo (Butorides striatus), Barraquete (Anas discors), Martín pe scador chico (Chloroceryle americana), Martín pescador enano (Chloroceryle aenea), Martín pescador matraquero (Chloroceryle amazona), Águila cienaguera (Busarellus nigricollis), Gavilán caminero (Buteo magnirostris), Garrapatero mayor (Crotophaga major), Laura (Catarthes aura), entre otros. En cuanto a los ma míferos, los grupos más abundantes son los murciélagos, sobre todo los frugívoros (Phillostomidae) y M urciélago común (Molossidae), Mono aullador (Alouatta seniculus) y Mico maic ero (Cebus apella). Igualmente el Chigüiro (Hydrochaeris hydrochaeris). Los reptiles están bien representados por la s especies Iguana (Iguana iguana), Icotea (Trachemys scripta callirostris), Lobito (Ameiva spp.) y La garto overo (Tupinambis teguixin). Por otra parte, los anfibios son abundantes, especialmente especies pertenecientes a la familia de las ranas Hylidae y Leptodactylidae, además de la Culebra de agua (Typhlo nectidae). En peces, a ctualmente se presenta una muy escasa población de Bocachico (Prochiludus magdalenense), pero $\mathrm{h}$ ay abundancia predominante de las especies Yalúa (Curimata magdalenae) y la Mojarra amarilla (Petenia kraussi). Para la flora, la especies arbóreas más abundante en los territorios de las ciénagas de Bañó y $L$ os Negros son el Espino (Pithecellobium lanceolatum), seguida del Dorado (Cassearia sp.), Naranjuelo (Crataeva tapia) y el Campano (Samanea saman). En cua nto a vegetaci ón acuática los espejos de agua en mención cuentan con Gramalote acuático (Hymenachne amplexicaulis), Bocachica (Thalia geniculata), Buchón (Eichhornea crassipes), Lechuguilla (Pistia stratiotes), Lenteja de agua (Salvinia auriculata), Trenza acuática (Paspalum repens), Tripa de mu erto (Neptunia prostrata), Oreja de $\mathrm{m}$ ulo (Eichhornea azurea), Torta (Nymphides humboldtianum), Clavito acuático (Jussiaea natans), Tabaquillo (Polygonum hydropiperoides), Najas (Najas argusta), Candelabro acuático (Ceratophyllum echinatum), Majate ( Utricularia foliosa), Cortadera (Cyperus luzulae), Paja co rtadera (Cyperus feraz), Mimosa (Mimosa somnians), Malva (Heliotropium indicum), Jazmín de los ríos (Cleome spinosa), Enea (Typha angustifolia), entre otros (Corporación de los Valle s del Sinú y San Jorg e, 2002).

Las ciénagas de Bañó y L os Negros presentan aguas lénticas, y su área está constituida fundamentalmente por zonas lacustres con un ciclo anual d e llenado y se quía. Aquí se pre sentan intercambios de agua con el río a través de un canal que comunica el río Sinú con I os humedales. Estas ciénagas reciben las aguas por dos vías: por crecientes del río Sinú, por medio del caño Concha y de la escorrentía de la cuenca aportante de la zona occidental a travé s del a rroyo El Polvero y en meno $r$ proporción por el arroyo El Tigre. 


\title{
Características socioeconómicas de la población residente en las áreas de los humedales de Bañó y Los Negros
}

\begin{abstract}
Las ciénagas de Bañó y Los Neg ros tienen como primeros vecinos a la s poblaciones de Cotocá Arriba, Palo de agua y Castilleral. Estas tres poblaciones son las que tienen mayor influencia en los territo rios de la s ciénagas, sin embargo se pueden mencionar como otros vecin os a las co munidades de Garavito, Guamal, Mompox y Saran delo, entre otras, las cuales ejercen presión sobre los humedales de manera esporádica. La población de los tres corregimientos se estima a 2014 en 3182 habitantes de los cuale s 2114 son a dultos $(66,4 \%), 557$ niños $(17,5 \%)$ y 489 adolescentes $(15,4 \%)$. Se observa que un $33 \%$ del total de la población se encuentra en un rango de edad de niño a adolescente, convirtiéndose en una fortaleza para la región si estos se llegasen a preparar para afrontar de forma eficiente un relevo generacional que contribuya de corto a mediano plazo a solucionar los problemas de orden ambiental que existen en el territorio.
\end{abstract}

En términos del tiempo de residencia de I os habitantes de la zona, se calcula que el $53,4 \%$ de las viviendas tienen al menos un individuo con más de 30 años de vivir en la zona de estudio y un $32,4 \%$ se encuentran en un rango de 16 a 30 años de habitar en la zona; si se suman ambos porcentajes y se tienen en cuenta los dos rangos, se estaría hablando de un $85,8 \%$ del total de la población que tiene 16 años o má $\mathrm{s}$ de vivir en el área, lo que indica que muchos de estos individuos han nacido, y crecido, en este territorio, además tienen un conocimiento amplio y empírico de los humedales y de los problemas presentes en ellos y cómo estos se han originado. Lo anterior contrasta con la estimación de que por lo menos el $58 \%$ de las viviendas encuestadas tienen un integrante con más de 40 años de edad (Figuras 2 y 3 ).

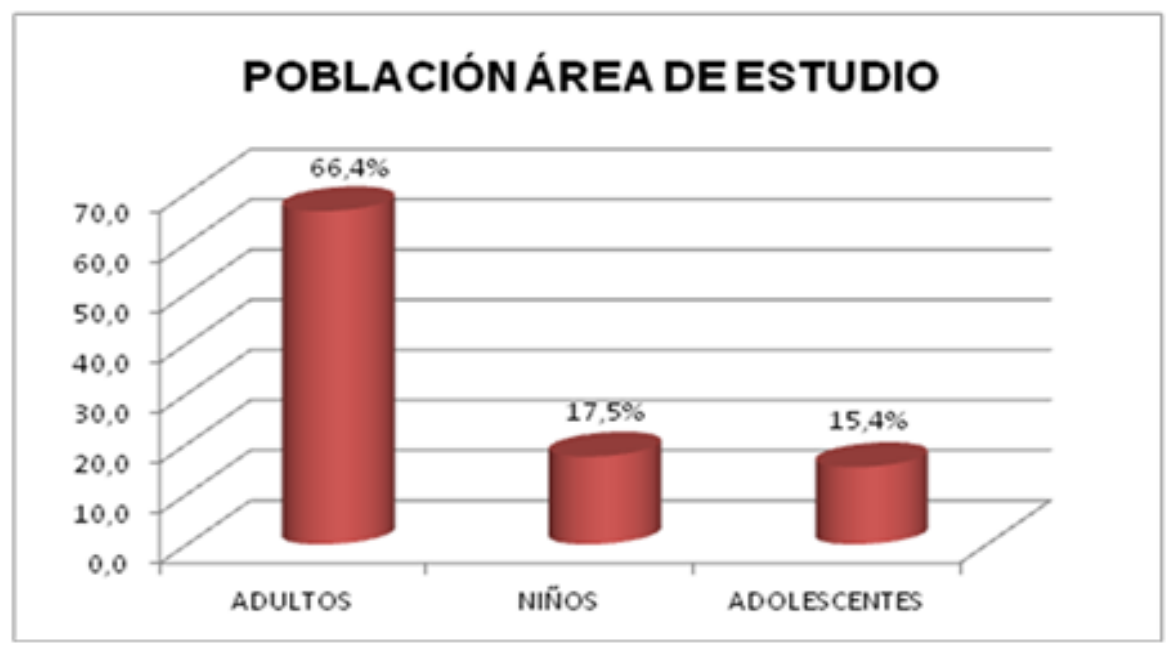

Figura 2. Distribución población del área de estudio 


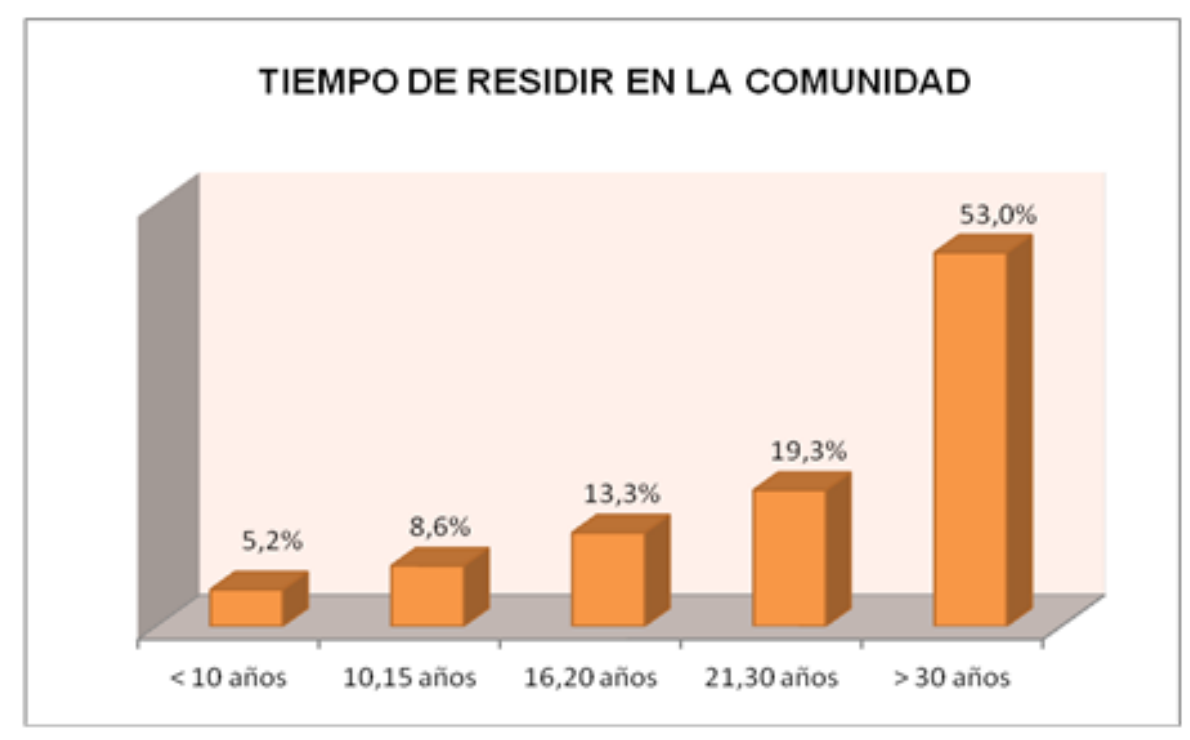

Figura 3. Tiempo de residencia en el área de estudio.

La educación es una de las variables que de manera similar al componente económico de las comunidades se categoriza en un nivel bajo. De las 645 viviendas habitadas se estima que 121 $(18,7 \%)$ cuentan con al menos un bachiller, $16(2,6 \%)$ con una persona iletrada, $98(15,1 \%)$ con al gún individuo con estudios técnicos, $115(17,7 \%)$ con estudios universitarios y $295(45,7 \%)$ con algún nivel de estudios básicos incompletos, sea primaria o secundaria. Si se suma el $45,7 \%$ de estudios básicos incompletos con el 2,6\% de personas iletradas, se hablaría de un $48,3 \%$ de vivienda $s$ encuestadas donde por lo menos un individuo no ha desarrollado de form a adecuada sus e studios básicos.

Se resalta que los mayores porcentajes de personas con estudios superiores pertenecen al corregimiento de Palo de Agua, territorialmente es el más grande de los tres y presenta mejor infraestructura educativa, se percibe de las personas mayor interés para educarse en comparación con los otros dos corregimientos, sin embargo lo anterior no quiere decir que los pobladores vecinos de la ciénaga no tengan un conocimiento profundo de estos e cosistemas ni d e la importa ncia que les representa para su supervivencia tanto a nivel indi vidual como comunitario. Así mismo, se estima que de la s 645 viviendas encuestadas al menos 419 tienen p or lo meno s un niño que asiste a la escuela, esto podría convertirse en una fortaleza para el territorio si los $p$ rocesos educativos se desarrollaran eficientemente (Figura 4). 


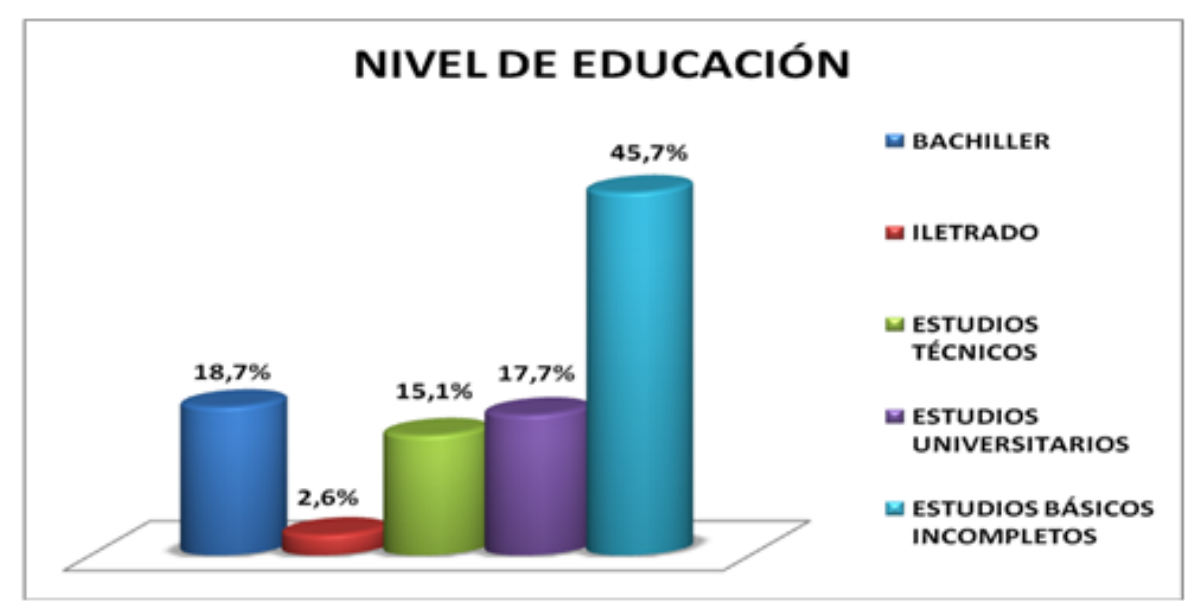

Figura 4. Nivel de educación de la población en el área de estudio.

En cuanto a la tenen cia de la tierra se calcula que en el 95,7\% (617) de I as viviendas residen sus propietarios, lo anterior se origina por un proc eso de títulos heredados de padres a hijos , excepto una pequeña porción del corregimiento de Castilleral, que en 1995 (dato suministrado por habitantes de la región) el gobierno otorgó parcelas con sus respectivos títulos de propiedad a familias qu e habían he cho posesión de terren os baldíos alrededor del corregimiento.

La infraestructura en servicios públicos es deficiente, aunque el $100 \%$ de las viviendas tienen fluido eléctrico, las tres poblaciones carecen de un relleno sanitario y de alcantarillado. Para el caso de Cotocá Arriba y Castilleral, solo cuentan con un tanque elevado que envía el a gua a I as casas sin ser potabilizada, solamente Palo de Agua cuenta con servicio de acueducto y sus aguas son tratadas y desinfectadas.

La infraestructura de las viviendas no es la más adecuada, las casas con mejor infraestructura cuentan con te chos construidos con tejas de asbesto cemento (material cancerígeno) y en el peor de los casos techo de palma o zinc. El 69,9\% (451) de las casas cuentan con un piso en cemento bruto o pulido, un $49 \%$ de las casas cuentan con dos habitaciones para familias que en promedio cuentan con 5 person as. El total de vivie ndas tiene al menos un televisor, un $70 \%$ posee refrigerador y tan solo un $13 \%$ (83) cuenta con un equipo de cómputo. La mayoría de I as viviendas cuentan con tan solo medio baño o ta za campesina (78\%). Se estima que en el $72 \%$ (467) de las vivien das habitan de 1 a 3 p ersonas por cuarto, en el re stante $28 \%$ (177) la habitabilidad está dada por un rango de 4 a 7 personas por habitación. Por las o bservaciones hechas en campo, en promedio habitan tres personas por alcoba.

En términos de hacin amiento hay que re saltar que existe $n$ diversos criterios para d efinirlo, variando según los diferentes países, sin embargo y por lo general cuando en un cuarto habitan más de 2,5 personas se considera que hay $\mathrm{h}$ acinamiento (Secretaría de Estado de Economía, Planificación y Desarrollo, 2007). Es claro, que cuando una vivienda presenta hacinamiento, 
sus ocupantes son vulnerables a sufrir ciertas problemáticas en el núcleo familiar, entre estas I a violencia doméstica, desintegración familiar, bajo re ndimiento escolar, entre otros (Comisión de Vivienda del Estado de Guanajuato, 2011).

Así mismo, 180 (28\%) viviendas habitadas al día de hoy utilizan fogón de leña, ocasionando presiones sobre el recurso boscoso, y posiblemente por el hecho de q ue todavía cocinen los alimentos con leña, el h umo que esta expide pu ede generar enfermedades pulmonares a las personas que están encargadas de la labor. Referente a la cobertura en salud en el territorio, se estima que el $82 \%(526)$ de las viviendas encuestadas cuenta con al menos un individuo afiliado al sistema de seguridad social otorgado por el Estad o o en su defecto por el Sistema de Identificación de Poten ciales Beneficiarios de Programas Sociales (SISBEN), siendo un porcentaje alto en cobertura.

A nivel d e organización comunitaria, existen varias organizaciones no gu bernamentales, dentro de estas se encuentran: Asopezca, Asopespa, Cotocá Viva, Asomupa, Fundación Bañó, entre o tras. El 34\% (222 ) de la s viviendas encuestadas cuentan con al menos un individuo que pertenece a alguna organización local y a pesa $r$ de existir o rganizaciones comunitarias, no hay un trabajo conjunto y articulado entre estas, cada una tiene sus propios objetivos y prioridades, no luchan en conjunto por sacar ad elante su territ orio sino que buscan el bienestar de unos pocos, sumiéndose aún más en el atraso y la pobreza mental y física. Para Bor dehore (1998) la capacidad de degradación ambiental varía según el modelo de orga nización social. Donde el ambient e forma pa rte de los val ores éticos, religiosos o culturales, el impacto a mbiental admitido por l a sociedad será mucho menor.

Se estima que de las 645 familias, 397 (62\%) tienen como actividad económica oficios varios, es decir, actividades como el mototaxismo, "un día de jornal", albañilería, reparaciones, "día de trabajo en el plancho", entre otras, son las que le brindan un ingreso económico a las familias, no siendo dichas labores bien remuneradas ni estables.

Llama mucho la atención que una región donde sus habitantes vivían de la pesca y la agricultura, en solo 16 años su actividad económica se haya reducido en un porcentaje tan alto, se estima que únicamente $62(10 \%)$ familias todavía practican la p esca como su principal actividad económica y prá cticamente la agricultura desapareció, muchos de los habitantes aseguran que ese cambio de a ctividad económica está asociado con la construcción de I a hidroeléctrica URRA (I a constitución de la Empresa Multipropósito de URRÁ S.A. se inició en 1992 y entró en operación en 2000), que trajo consigo efectos adversos al río Sinú y los humedales aledaños, entre estos Bañó y Los Negros. Actividades económicas relacionadas con el comercio (13\%), la ganadería $(6 \%)$ la do cencia $(5 \%)$ y la minería de materiales de arrastre $(3 \%)$ se convierten en ot ras de las "o pciones" para la generación de ingresos en los corregimientos vecinos a lo s humedales (Figura 5). 


\section{ACTIVIDAD ECONÓMICA EN LA ZONA}

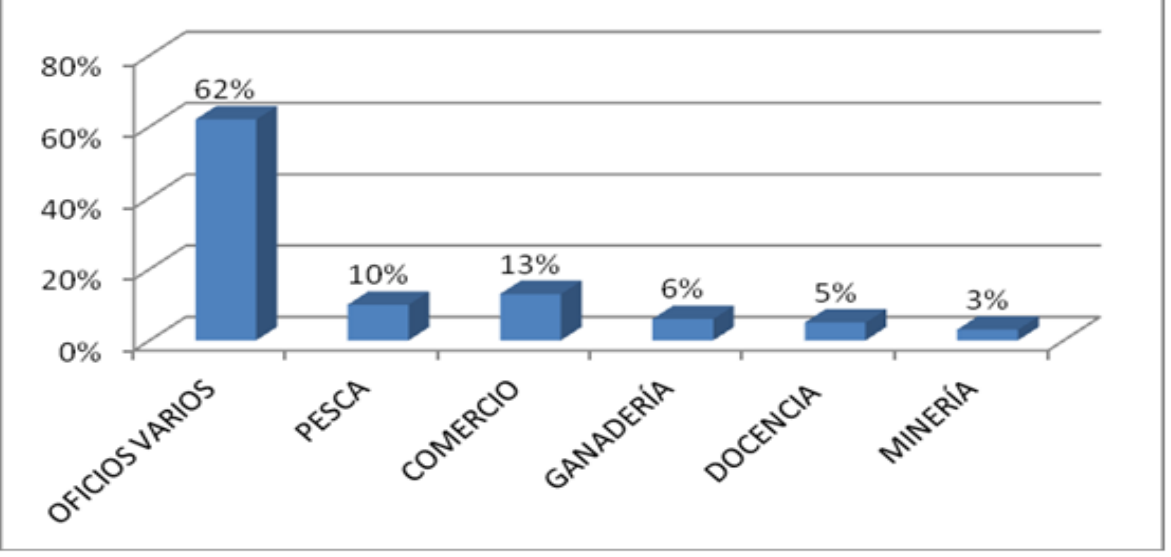

Figura 5. Actividad económica en la zona de estudio.

Básicamente, 282 (44\%) familias obtienen sus ingresos diarios y un 33\% (212) semanalmente. Un 53\% (342) de las familias en el territorio reciben ingresos diarios menores a $\$ 10.000$ (un poco más de US\$5, se po dría decir que e n promedio reciben entre $\$ 5.000$ a $\$ 7.000$ por familia), 204 (32\%) familias tienen ingresos diarios en un rango de $\$ 10.000$ a menos de $\$ 20.550$ (un poco más de US\$5 y menos de US\$11, en promedio $\$ 15.000$ diarios). El $85 \%$ de I as familias asentadas alrededor de los humedales tienen ingresos económicos muy b ajos, ingresos que se categorizan en la línea de pobreza y pobreza extrema si se compara con los valores proporcionados por el Departamento Nacional de Estadística (DANE, 2012) en su boletí n de pobreza monetaria y multidimen sional en Colombia, donde clasifica de acuerdo a valores de ingreso por familia que un hogar compuesto por 4 personas será clasificado como pobre si el ingreso total del hogar está por debajo de \$808.332 (US\$429,96). Si la familia vive en las cabeceras este valor cambia a $\$ 892.604$; si vive en el área rural a $\$ 534.088$. En términos de la pobreza extrema si un hogar está compuesto en promedio por 4 personas, se clasificarán como pobres extremos los hogares en donde su ingreso total esté po $r$ debajo de $\$ 364.828$. A $n$ ivel de las cabeceras este valor cambia a $\$ 381.404$, en área rural el valor es de $\$ 310.880$.

Es difícil que con tan baj os ingresos alcancen a sobrevivir I as familias aledañas a las ciénagas, sobre todo cuando las mismas aseguran que el volume $\mathrm{n}$ de in gresos no les permite vivir dignamente y cuando en promedio hay núcleos familiares de 5 personas. Se estaría hablando de una "economía del rebusque". Paralelo a esto, 408 (63\%) familias reciben ayuda económica por parte del gobierno, concretándose en el conocido programa Familias en Acción promovido por el Departamento para la Prosperidad Social, el cual reali za transferencias monetarias condicionadas a familias pobres, otorgando a los menores de edad que integran el nú cleo familiar un "incentivo económico bimensual" que oscila entre los $\$ 25.000$ a $\$ 57.000$ (US\$12,5 a US\$28,5) dependiendo del grado académico que curse el educando. En palabras de la líd er comunitaria y Mujer CAFAM 
2009, Purificación del Carmen Sánchez, esto se constituye en "La limosna para enseñar a ser limo sneros". ¿Qué va a pasar cuando el Estado ya no pueda dar más este tipo de subsidios?

Se tiene una población con bajos recursos económicos y pocas oportunidades de mejorar su calidad de vida, y cada vez ejerce mayor presión sobre los humedales, que son la úni ca fuente de recursos naturales con que cuenta (Figura 6). Para Boy ce (citado por Correa, 2 007) las desigualdades sociales y económicas son factores determinantes en la explicación de la calidad ambiental.

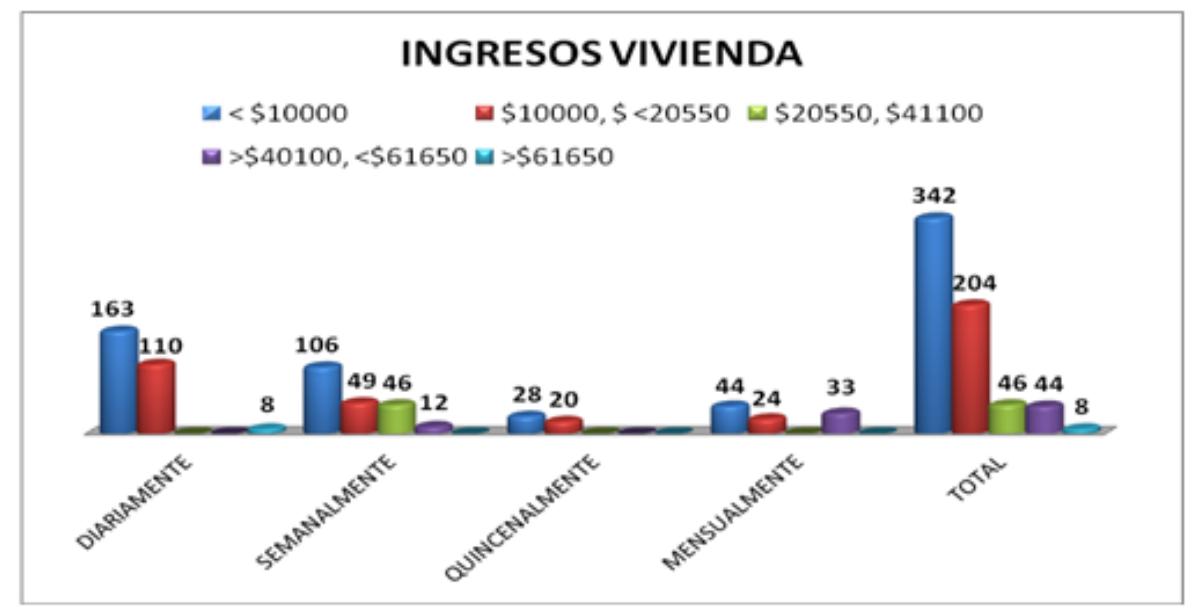

Figura 6. Ingresos por nucleo familiar en la zona de estudio.

\section{Los humedales de Bañó y Los Negros: problemática ambiental y su importancia ecológica}

Las ciénagas de Bañ ó y Los Negros son h umedales de tipo ribereño, se encuentran bordeando el cau ce del río Sinú. Al principio ambos espejos de agua eran un solo cuerpo, pero en 1965 (el año es aproximado, este dato fue suministrado por los habitantes que tienen el mayor tiempo de residir en el lugar) se construyó la carretera para interconectar los corregimientos de Cotocá Arriba, Palo de Agua, Castilleral entre otros. Lo anterior trajo consigo la división y morfometría que actualmente poseen. Como respuesta para mantener el equilib rio hidráulico de los espejos de agua se implementaron unas estructuras conocidas como "puentes box culvert" que permiten el flujo y reflujo entre las ciénagas. Además, $\mathrm{I}$ os humedales en $\mathrm{m}$ ención prestan servicios ambientales a sus habitantes, cumplen con una serie de funciones ecológicas en el territorio, ofrecen recursos naturales para su supervivencia y sirven de sumidero para los residuos que estos generan. La importancia de este tipo de ecosistemas en la región del bajo Sinú radica principalmente en constituirse en un reservorio de agua natural, regulador del microclima en su áre a de influencia, de inun daciones y sequías, de hábitat para diversas especies de fauna y flora y de rel evancia para el fomento de una cultura alrededor de los mismos. 
Por la impo rtancia que tienen los humedales de Bañó y Lo s Negros, como hábitat de muchas especies de aves migratorias y endémicas, se han declarado recientemente Áreas de Importancia para la Conservación de Aves (AICA). S e encuentran reportadas 49 especies de aves acu áticas, de las cuales nueve son migratorias y 34 son residentes (Arzuza, Moreno y Salaman, 2 008). Las Áreas de Impo rtancia para la Conservación de las Ave s (AICAS) son una ini ciativa que se promueve a nivel mundial para la id entificación y declaratoria de hábitat de aves amenazadas de extinción a niv el global o nacional, sitios de endemismos o congregación de especies, como zonas de especial importancia para adelantar acciones en conservación e inve stigación sobre riqueza y estado de poblaciones de avifauna (Instituto A lexander von Humboldt, 2003). Por otra pa rte, los hum edales configuran importantes reservas de diversidad biológica y su alta $p$ roductividad da sustento a una gran riqueza y abundancia de especies. Más del $40 \%$ de las especies del mundo y el $12 \%$ de to das las especies animales se hallan en lo $s$ humedales de agua dulce. Las zonas húmedas son además un importa nte reservorio genético (Corporación Ambiental de Acuáticos de Chile, 2005, p. 58).

En el área de influencia de los humedales se observa una serie de limitantes del territorio que no cont ribuyen a la conservación de los mismos, señalándose: a) Centralización de la propiedad de la tierra, b) Deterioro de ecosistemas presentes en el territorio, c) Altos niveles de pobreza y miseria de la población, d) Pocas fuentes de empleo, e) Baja infrae structura vial, saneamiento básico y poca cobe rtura en se rvicios públicos, f) Bajo nivel de educación y conci encia ambiental, g) Subvalo ración de la $s$ potencialidades ambientales y h) Po ca capacidad de gestión y articulación institucional. De igual forma, las presiones a las que se ven sometidos ambos espejos por parte de las comunidades vecinas son constantes.

Los problemas ambientales dependiendo de su origen se clasifican en naturales y antrópicos. Los problemas ambientales de tipo antrópico son el resultado de la fo rma en que las sociedades toman los elementos que necesitan de la naturaleza, transformándola para generar bienes y servicios. Básicamente los problemas ambientales de Bañó y Los Negros se resumen en los siguientes (ver matriz detallada en la Tabla 1):

1. Desecación de los humedales: Producto de la construcción de terraplenes por parte de terceros para cortar los flujos de agua y generar procesos de sedimentación, con el fin de co nstruir potreros para pastar el ganado y $\mathrm{p}$ oder realizar cultivos $\mathrm{d}$ e pancoger, dando origen a conflictos de uso de suelos, sin omitir el mal manejo que se le da a la compuerta que se encuentra ubicada en el municipio de San Pelayo, la cual co rta el flujo de agua hacia los humedales, además de las fuertes sequías originadas por el cambio climático.

2. Pérdida de la biodiversidad: Factores como la ca za indiscriminada de espe cies como Iguana (Iguana iguana), Chigüiro (Hydrochaeris hydrochaeris), Icotea (Trachemys callirostris), Babilla (Caiman fuscus), Perico (Aratinga holochlora brevipes), entre otras, especialmente en lo $\mathrm{s}$ periodos de la 
Semana Mayor, la tala de árboles para la elaboración de cercas y leña para cocinar, fragmentación de los ecosistemas por la construcción de la carretera Cotocá Arriba-Castilleral, sobrepastoreo delos terrenos de la ciénaga, la débil cultura ambiental, el cambio climático, la introdu cción de especies foráneas que desplazan a ot ras especies autóctonas apoderándose del te rritorio (Tilapia), la quema de los bordes de las ciénagas, manejo irracio nal del recurso pesquero, han contribuido al deterioro de la diversidad biológica en la región.

3. Contaminación y deterioro del recurso suelo y agua: El uso inadecuado de pe sticidas derivados de las actividades ganaderas, así como el v ertimiento tanto en el suelo como al agua de tales residuos, la quema de los suelos y su mal manejo, la débil cultura del respeto por la natu raleza y el poco control por parte de las autoridades ambientales, han tenido com o consecuencia la contaminación y deterioro de los valiosos recursos: suelo y agua.

4. Manejo inadecuado de residuos: La ausencia de un sitio para la correcta disposición de residuos sólidos y l íquidos, el desconocimiento para el manejo adecuado de residuos por parte de los habitantes y la po ca gestión de las autoridades locales y ambientales, han generado el escenario para la a cumulación de residuos en los corregimientos y la aparición de vectores. Sin dejar de lado que los espejos de agua cumplen una función de sumidero para la recepción de los residuos sólidos y líqui dos provenientes de algunas casas debido a la $s$ deficientes condiciones sanitarias, y la au sencia de una cultura ambiental que contribuya a gene rar acciones positivas hacia el medio por parte de los pobladores del área de influencia.

5. Deficientes condiciones sanitarias: La construcción de po zas sépticas ineficientes, la ause ncia de un sistema de alcantarillado y acueducto a nivel ru ral, la falta de $p$ lanificación territorial y la poca gestión por parte de las autoridades locales y ambientales, han generado el escena rio para que en los corre gimientos no existan condiciones sanitarias adecuadas. 
Tabla 1. Matriz de problemas ambientales de las ciénagas de Bañó y Los Negros

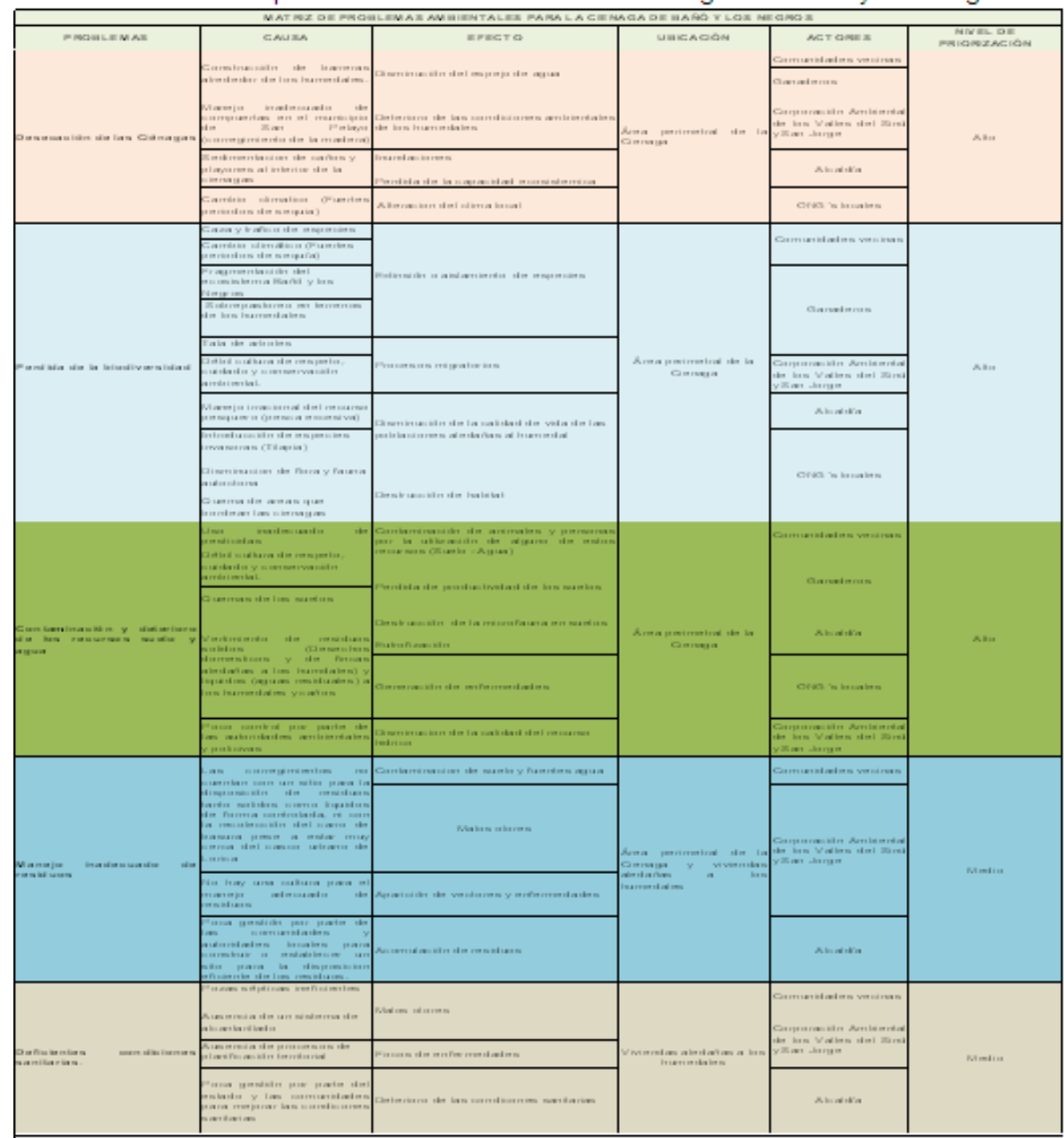

Hacer click sobre la imagen para ampliarla

González y Valencia (2013) señalan que el interés por los problemas ambientales surge a raíz del grado de destrucción ambiental de la atmósfera y de los recursos naturales, lo anterior ha generado un pro ceso de encareci miento de lo s productos naturales y una crisis ambiental, igualmente los problem as ambientales avanzan a d iario, la ca pacidad de re spuesta del hombre frente a ellos ha quedado rezagada, y evidentemente los conflictos generados por las carencias generalizadas (guerras del agua, desaparición de los bosques, desertificación de la Tie rra, aglomeración humana, hambre) se han acentuado y extendido.

En el mismo orden de ideas, al preguntarle a los habitantes de las ciénagas cuáles eran para ellos los principales problemas ambientales que los afectaban, consideraron que la desecación de los humedales, las deficientes condiciones sanitarias, la poca cultura ambiental, el ma nejo irracional de re siduos, la caza y tráfico de especies, la falta de espaci os recreativos, y la sedimentación y tapon amiento de los caños, se constituyen en causas para el deterioro de las condiciones ambientales del área de estudio. Por lo tanto, las comunidades tienen nociones de los problemas que los afectan y no están muy distantes de I os resultados consignados en la matriz de problemas ambientales, 
obtenida de información proporcionada por estudios ambientales realizados en la zona y del trabajo de campo para la confirmación de la información secundaria suministrada por estos. Se resalta que las $\mathrm{p}$ oblaciones aledañas a los humedales sí tienen un conocimiento, aunque sea de carácter empírico, de su há bitat o entorno.

De igual modo, en los procesos de gestión ambiental pueden intervenir diversos actores.

Los actores de la gestión ambiental son aquellos grupos de la soci edad que id entifican la problemática del medio ambiente, y que formulan e implementan políticas dirigidas a su protección. Se consideran actores claves del a gestión ambiental a: las orga nizaciones gubernamentales, los grupos organizados de la sociedad civil, los me dios de comunicación masiva, y las empresas del sector privado que se han organizado explícitamente en $\mathrm{p}$ ro de la protección ambiental. (Rodríguez y Espinosa, 2003, p. 10)

Para el caso de los humedales de Bañó y Los Negros, actores como las co munidades, ganaderos, la administración local y la Corporación Ambiental de los Valles del Sinú y San Jorge (CVS) son fundamentales en la solución de la problemática ambiental. Es necesario desarrollar un trabajo articulado entre cada uno de los actores y asumir sus roles y fu nciones correspondientes. Problemas ambientales como la pérdida de la biodiversidad, la desecación de los humedales y la contaminación del re curso suelo y agua tienen un nivel de priorid ad alto en la búsque da de soluciones debido a que los impactos de tipo negativo generados han ido transformando y deteriorando los ecosistemas. El deseo de los ganaderos por tener más tierras los ha impulsado a secar los bordes de las ci énagas con fines de pasto reo alterando el equilibrio ecológico y disminuyend o la calidad am biental en I a zona. Hoy e $\mathrm{n}$ día, debid o al dete rioro ambiental del área de estudio, se encuentra comprometida la seguridad alimentaria de sus habitantes, el deterioro de los suelos por el mal manejo de los mismos ha disminuido su capacidad productiva, asociado a esto el cambio climático ha acelerado su deterioro.

\section{CONCLUSIONES}

Para la g eneración de un modelo educativo no fo rmal para la conservación con fines de protección de los humedales de Bañó y Los Negros a partir de la valoración ambiental del territorio se concluye lo siguiente:

- Factores como los bajos niveles de ingresos económicos, pocas oportunidades de empleo, bajo niv el educativo, deficientes condiciones sanitarias, están contribuyendo al deterioro de los humedales. Existe poca conciencia por la p rotección de lo s 
humedales; actividades como la caza indiscriminada, la pesca sin control de tallas, los vertimientos de contaminantes a los cuerpos de agua y suelos, las quemas de las áreas que bordean las ciénagas, entre otros más, son indicadores de la inadecuada interacción de los habitantes con los respectivos ecosistemas.

- Las comunidades a p esar de e star organizadas no procuran trabajar de forma articulada y bajo los mismo s intereses y objetivos, siendo la in dividualidad y el interés particular los que priman sobre el bienestar común. Es necesario que las comunidades desarrollen un verdadero trabajo com unitario que propenda por mejorar la calidad de vida de I as mismas y da $r$ solución a la problemática ambiental existente.

- Es probable que de no mejorar las condiciones socioeconómicas de las po blaciones aledañas a los humedal es se gene ren procesos migratorios hacia los pri ncipales centros poblados, y que aquellos habitantes que termi nen desplazándose a di chos centros contribuyan a engrosar los cordones de miseria e inseguridad de los centros urbanos debido a su baja preparación y recursos económicos.

- Actores como las comu nidades, ganaderos, la Corp oración Autónoma Regional de los Valles del Sinú y San Jorge (CVS), la Alcaldía de Lorica y las ONG presentes en el territorio, son claves en la búsqueda de soluciones a los problemas ambientales de los humedales.

- Las poblaciones vecinas a los humedales tienen habitantes con más de 16 años de residir en el te rritorio, convirtiéndose en una fortaleza ya que poseen un conocimiento muy profundo de este tipo de eco sistemas, a pesar no tener u na buena relación hombre-medio.

- Las comunidades no poseen una visión holística que les ayude a comprender de forma sistémica la importancia que tienen ambos espejos de agua para la supervivencia de las mismas.

- Los humedales de Bañó y Lo s Negros prestan servicios ambientales relevantes a la comunidad al ofrecer recursos a sus pobladores.

\section{RECOMENDACIONES}

A partir de la problemática ambiental detectada en el diagnóstico realizado en la zona de influencia del presente estudio se presentan las siguientes recomendaciones:

- Generar un modelo educativo no formal qu e responda a la s particularidades del territorio y del cual se logren empoderar las comunidades residentes aledañas a los humedal es, convirtiéndose dicho modelo en un pilar para el desarrollo de las mismas.

- Vincular la capacitación ambiental con proyectos de autogestión de organizaciones comunitarias.

- Comprometer a las aut oridades locales y re gionales con programas de desarrollo sostenible.

- Implementar proyectos de seguimiento y evalua ción de dichos proyectos y programas. 


\section{REFERENCIAS}

- Alcaldía de Lorica. (2001). Plan de Ordenamiento Territorial del Municipio de Santa Cruz de Lorica. Secretaría M unicipal de Planeación. Lorica, Córdoba.

- Arzuza, E., Moreno, I. y Salaman, P. (2008). Conservación de las aves acuáticas en Colombia. Proaves. Recuperado de http://www.proaves.org/wp-

content/uploads/2008/12/C.Colombia6.Nov25Logo.pdf

- Bindé, J. (2008). El porvenir de la Tierra ¿Qué futuro para la humanidad? Política Exterior, 123, 103-112.

- Bordehore, C. (199 8). Problemas ambientales, problemas humanos. Rua. Recuperado de http://rua.ua.es/dspace/bitstream/10045/2725/8/cap8.pdf

- Capra, F. (2003 ). Conexiones ocultas. Barcelona: editorial Anagrama.

- Comisión de Vivienda d el Estado de Guanajuato (COVEG). (2011). Sistema de Indicadores de Vivienda. Re cuperado de https://www.coveg.gob.mx/seiisv/modulos/secciones/ publicaciones/Sistema_indicadores_gto.pdf

- Corporación Ambiental de Acuáticos de Chile. (2005). Manual para el uso Racional de sistemas costeros de Coquimbo. Centrotropical.org. Recuperado http://www.centroneotropical.org/recsos/manual_01.pdf

- Corporación de los Valles del Sinú y San Jorge (CVS). (2002). Caracterización de Flora y Fauna en Ciénaga de Bañó, Municipio de Lorica - informe final.

- Correa, F. (2007). Crecimiento económico, desigualdad social y medio ambiente: evidencia empírica para Amé rica Latina. Ingenierías, $6(1)$ Recuperado de http://www.scielo.org.co/scielo.php?script=sci_arttext\&pid= S1692-33242007000100002

- Departamento Nacional de Estadística (DANE). (2012). Boletín de Pobreza Monetaria y Multidimensional en Colombia. Recuperado de

https://www.dane.gov.co/files/investigaciones/condiciones_vi dal pobreza/boletin_pobreza_2012xx.pdf

- González, F. y Va lencia, J. (2013). Conceptos básicos para repensar la problemática ambiental . Gestión y Amb iente, 16(2), 121-128.

Recuperado

de http://www.redalyc.org/pdf/1694/169428420010.pdf

- Instituto de Investiga ción en Recursos Biológicos Alexander von Humboldt. (2003). Documentos AICA's Colombia. Recuperado de http://www.humboldt.org.co/humboldt/mostrarpagina.php?co dpage $=3000017$

- Instituto Geográfico Agustín Codazzi (IGAC). (1983). Estudio general de los suelos de los muni cipios que conforman la parte media y baja de la cuenca del Río Sinú.

- Meira, A. (2006). Crisis ambiental y globalización: Una le ctura para educadores ambientales en un mun do insostenible. Trayectorias, VIII, 110-1 23. Recuperado de http://www.redalyc.org/articulo.oa?id=60715248011 
- Rangel, J., Lowy, D. y Aguilar, M. (1997). Distribución de los tipos de vegetación en las regiones naturales de Colombia. En Colombia Diversidad Biótica II (pp. 383-402). Bogotá: Universidad Nacional de Colombia.

- Rodríguez, B. y Espino sa, G. (200 3). Gestión Ambiental e n América Latina y el Caribe: Evolución, tendencias y principales prácticas. Recuperado de http://www.iadlb.org/sds/publication/publication_3351_s.htm

- Secretaría de Estado de Economía, Planificación y De sarrollo. (2007). Hacinamiento en la Rep ública Dominicana. Recuperado de

http://www.one.gob.do/index.php? module=uploads\&func=do wnload

1. MSc. en Gestión Ambiental. Fundación Salud Mental para Todos. Departamento de Córdoba. Montería, Colombia. villalord15@yahoo.es

2. Doctor en Pedagogía. Instituto Tecnológico de Costa Rica. Cartago, Costa Rica. d_huffman@yahoo.com

3. Profesional en Estadística. Fundación Salud Mental para Todos. Departamento de Córdoba. Montería, Colombia. guerrerostalyn@hotmail.com

4. MSc. en Desa rrollo Rural. CORPOICA. Departamento de Cundinamarca, Bogotá, Colombia. tatiana.rivero@gmail.com

5. MSc. en Gestión Ambiental. Fundación Kontiqui. Departamento de Bolívar. Ca rtagena de Indias, Colombia. acobo71@yahoo.com

Para citar este artículo: Villadiego Lorduy, J. , HuffmanSchwocho, D., Guerrero Gómez, S., Rivero Espitia, S. y Cortecero Bossio, A. (2 015). Valoración ambiental para la generación de un modelo participativo de educación no formal. Revista Luna Azul, 41, 165-183. Recuperado de http://lunazul.ucaldas.edu.co/index.php?option=content\&tas k=view\&id=1061 